\title{
The OLYMPUS Experiment - Two-Photon Exchange in Electron Proton Scattering
}

Outline

$>$ Introduction and Motivation

$>$ Overview of the Experiment

$>$ Radiative Corrections

$>$ Results Uwe Schneekloth, DESY

$>$ Conclusions

Lomonosov Conference2017 Moscow OLYMPUS Collaboration 


\section{Elastic e N Scattering/Form Factors}

Nucleon elastic form factors: electric $G_{E}$ and magnetic $G_{M}$

$>$ Fundamental observables describing distribution of charge and magnetism in proton and neutron

$>$ Described by quark structure of proton

$>$ Will be calculable in lattice QCD

$>$ For $\sim 50$ years unpolarized cross section measurements have determined $G^{p}$ and $G_{M}^{p}$ using the Rosenbluth separation

$$
\begin{array}{ll}
\frac{d \sigma / d \Omega}{(d \sigma / d \Omega)_{M o t t}}=\frac{\sigma}{\sigma_{0}}=A\left(Q^{2}\right)+B\left(Q^{2}\right) \tan ^{2} \frac{\theta}{2} & \sigma_{\text {red }}=\frac{d \sigma}{d \Omega} \frac{\varepsilon(1+\tau)}{\sigma_{M o t t}}=\tau G_{M}^{2}+\varepsilon G_{E}^{2} \\
=\frac{G_{E}^{2}\left(Q^{2}\right)+\tau G_{M}^{2}\left(Q^{2}\right)}{1+\tau}+2 \tau G_{M}^{2}\left(Q^{2}\right) \tan ^{2} \frac{\theta}{2} & \tau=Q^{2} / 4 M_{p}^{2} \quad \varepsilon=\left[1+2(1+\tau) \tan ^{2} \theta / 2\right]^{-1}
\end{array}
$$

( $\varepsilon$ transverse virtual photon polarization) 


\section{Form Factors - Rosenbluth Method}

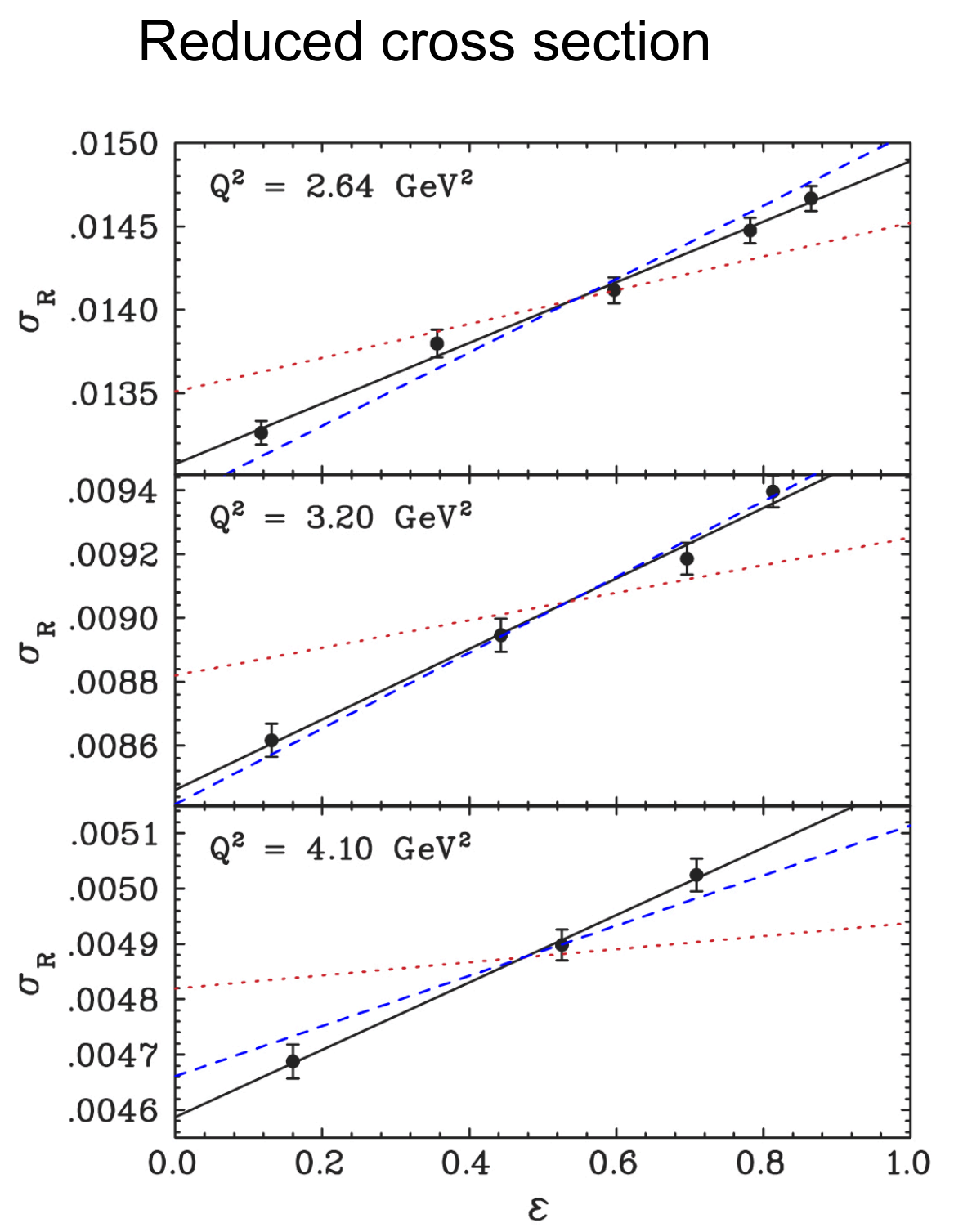

$$
\sigma_{r e d}=\frac{d \sigma}{d \Omega} \frac{\varepsilon(1+\tau)}{\sigma_{M o t t}}=\tau G_{M}^{2}+\varepsilon G_{E}^{2}
$$

Vary $E$ and $\theta$ to measure $\sigma_{R}$ different $\varepsilon$ but same $Q^{2}$ and plot:

$>$ Slope $\rightarrow G_{E}^{2}$

$>$ Intercept $\rightarrow G_{M}{ }^{2}$

$>G_{M}$ dominates at high $Q^{2}$

$>\sigma_{R}$ decreases quickly with $Q^{2}$

Blue dashed: $\mathrm{FF}$ ratio $=1$

Red dotted: pol. measurements

I.A. Qattan, Phys. Rev. Lett. 94 (2005) 142301. 


\section{Discrepancy in Form Factor Ratio}

Proton Form Factor Ratio vs. $Q^{2}$

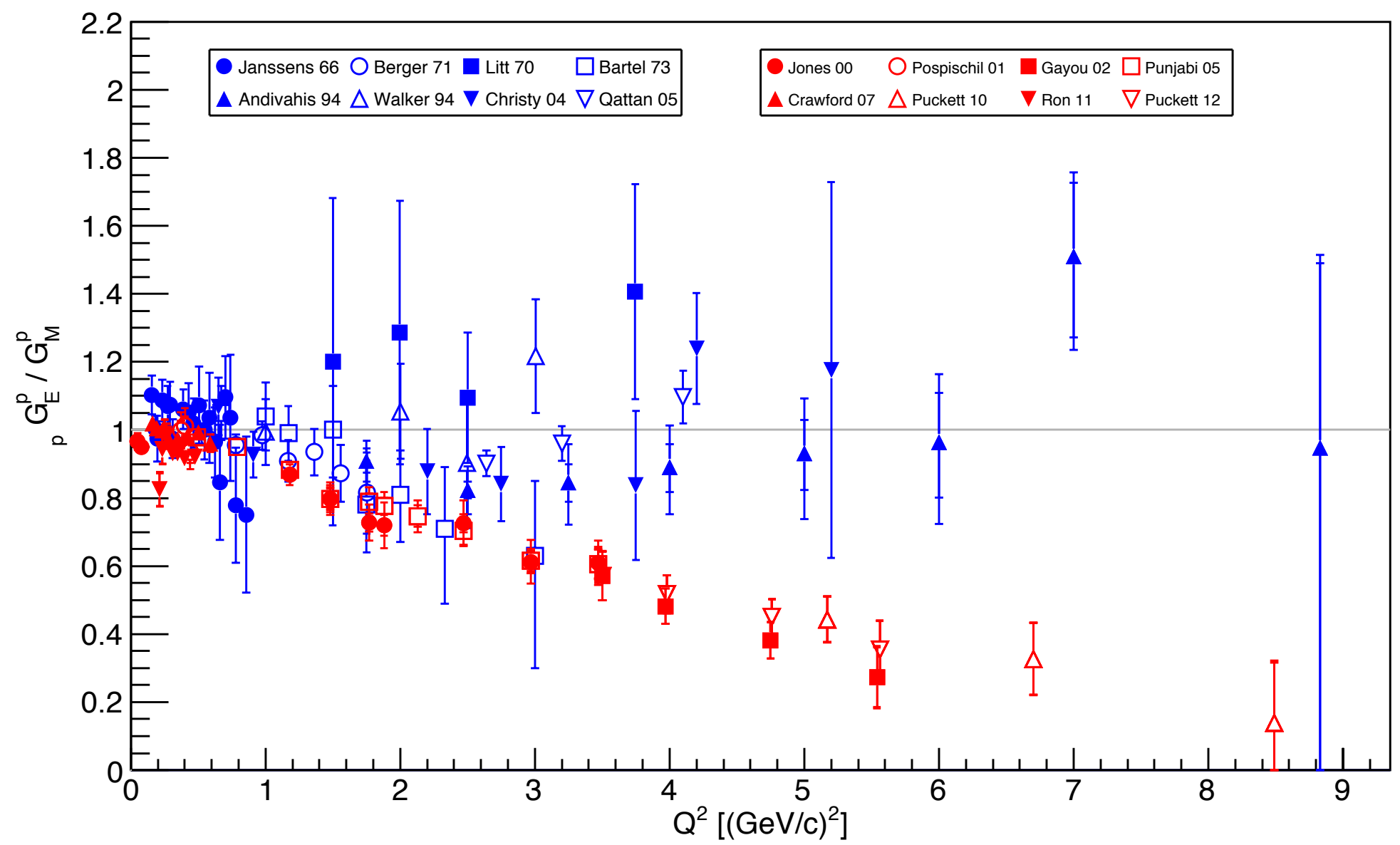

$>\quad$ All Rosenbluth data in agreement

$>$ Dramatic discrepancy between Rosenbluth and recoil polarization technique

$$
\begin{aligned}
& \vec{e} p \rightarrow e \vec{p} \\
& \mu_{p} \frac{G_{E}}{G_{M}}=-\mu_{p} \sqrt{\frac{\varepsilon(1+\varepsilon)}{2 \varepsilon}} \frac{P_{T}}{P_{L}}
\end{aligned}
$$

$>\quad$ Interpreted as evidence for two photon contribution to elastic scattering 


\section{Proposed Explanation - Two Photon Exchange}

\section{Two-Photon-Exchange}

$>$ Thought to be small effect

- Suppressed by order $\alpha$

$>$ Hard TPE difficult to calculate

- Intermediate $p, \Delta, \ldots$

- Large theoretical model uncertainties

$>$ Calculations suggest TPE can resolve discrepancy

$>$ Only experiment can definitively resolve contributions beyond single photon exchange

$>$ Determine TPE by measuring ratio of $e^{+} p / e^{-p}$, i.e. ratio of rates, no absolute cross section measurements
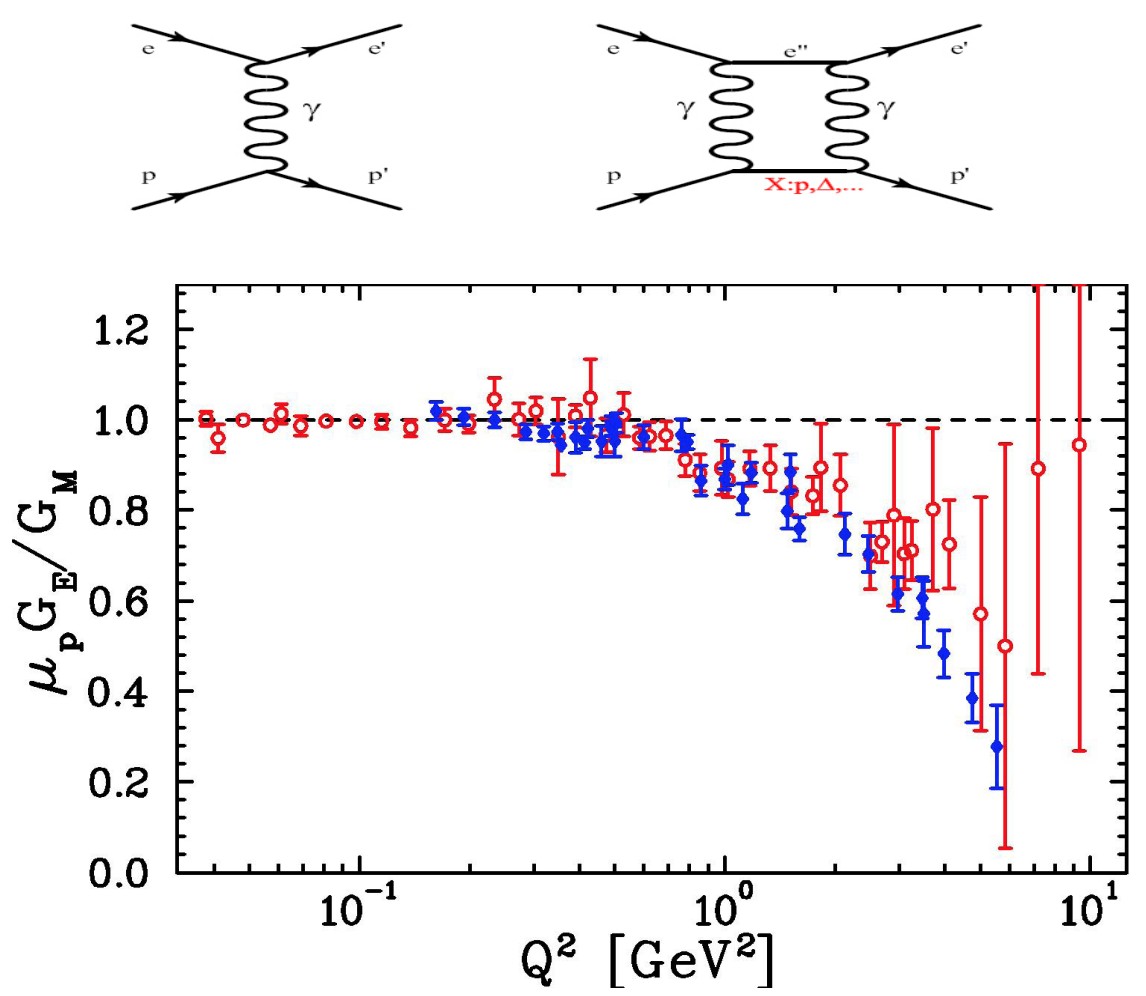

J. Arrington, W. Melnitchouk, J.A. Tjon, Phys. Rev. C 76 (2007)

$$
\begin{aligned}
\sigma\left(e^{-} p\right) & =\left|M_{1 \gamma}\right|^{2} \alpha^{2}-2\left|M_{1 \gamma}\right|\left|M_{2 \gamma}\right| \alpha^{3}+\ldots \\
\sigma\left(e^{+} p\right) & =\left|M_{1 \gamma}\right|^{2} \alpha^{2}+2\left|M_{1 \gamma}\right|\left|M_{2 \gamma}\right| \alpha^{3}+\ldots \\
R & =\frac{\sigma\left(e^{+} p\right)}{\sigma\left(e^{-} p\right)}=1+\frac{4 \Re\left(M_{1 \gamma}^{\dagger} M_{2 \gamma}\right)}{\left|M_{1 \gamma}\right|^{2}}
\end{aligned}
$$




\section{OLYMPUS Experiment at DORIS}

Elastic $\mathrm{e}^{+}\left(\mathrm{e}^{-}\right) \mathrm{p}$ scattering at $2 \mathrm{GeV}$ beam energy

$>$ Measure ratio of $e^{+} p / e^{-p}$ rates with $1 \%$ precision

$>$ DORIS $100 \mathrm{~mA} \mathrm{e}^{+}\left(\mathrm{e}^{-}\right)$beam

$>$ Unpolarized internal hydrogen target, density $3 \times 10^{15} \mathrm{at} / \mathrm{cm}^{2}$

$>$ Daily change of beam ( $\mathrm{e}^{+}$or $\left.\mathrm{e}^{-}\right)$to minimize systematic error

$>$ Redundant luminosity measurements

$>$ Using former BLAST detector from MIT/ Bates. Ideally suited.

Comparison of data and theory

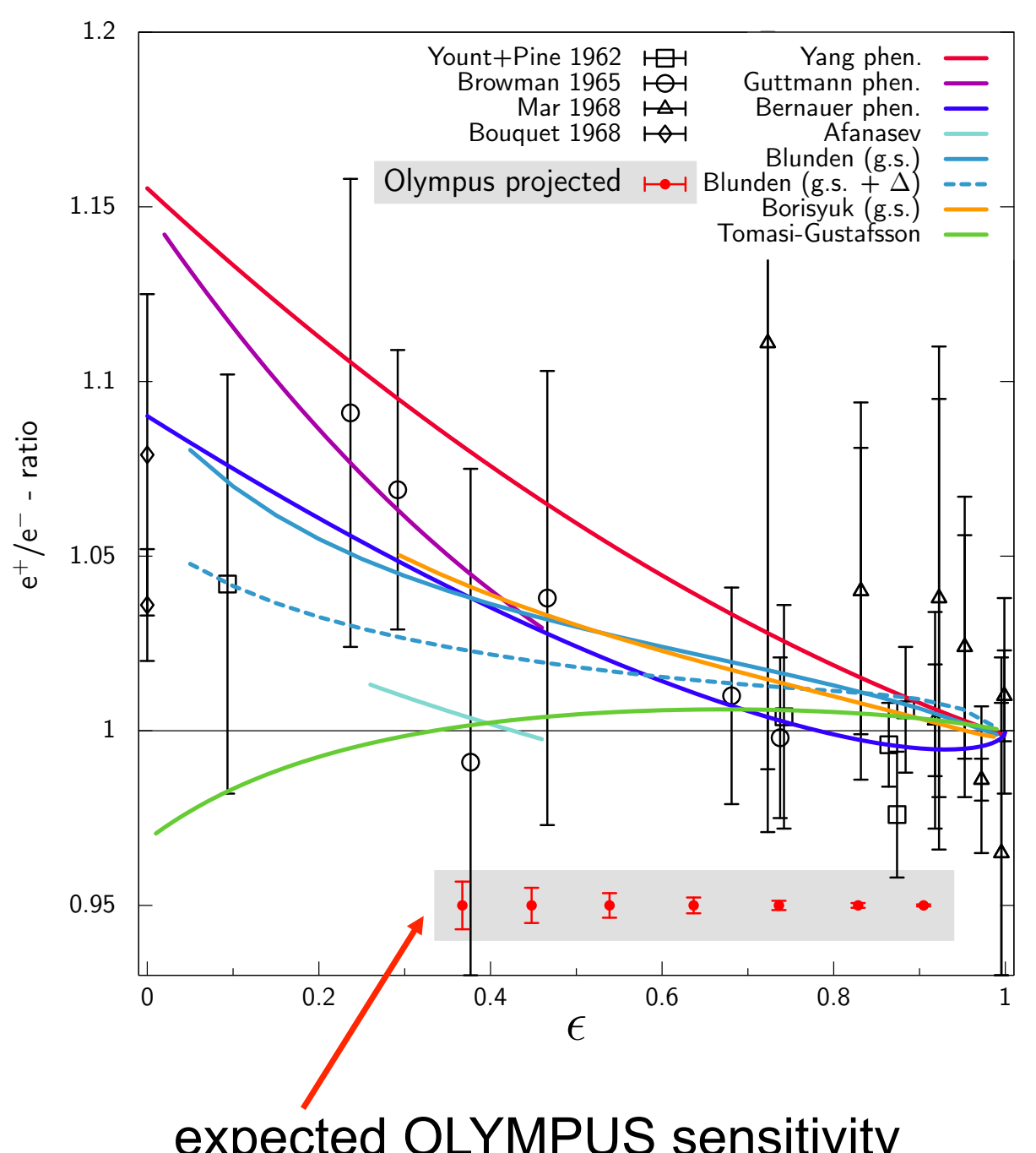

OL $\leqslant$ MPUS 


\section{Detector Overview}

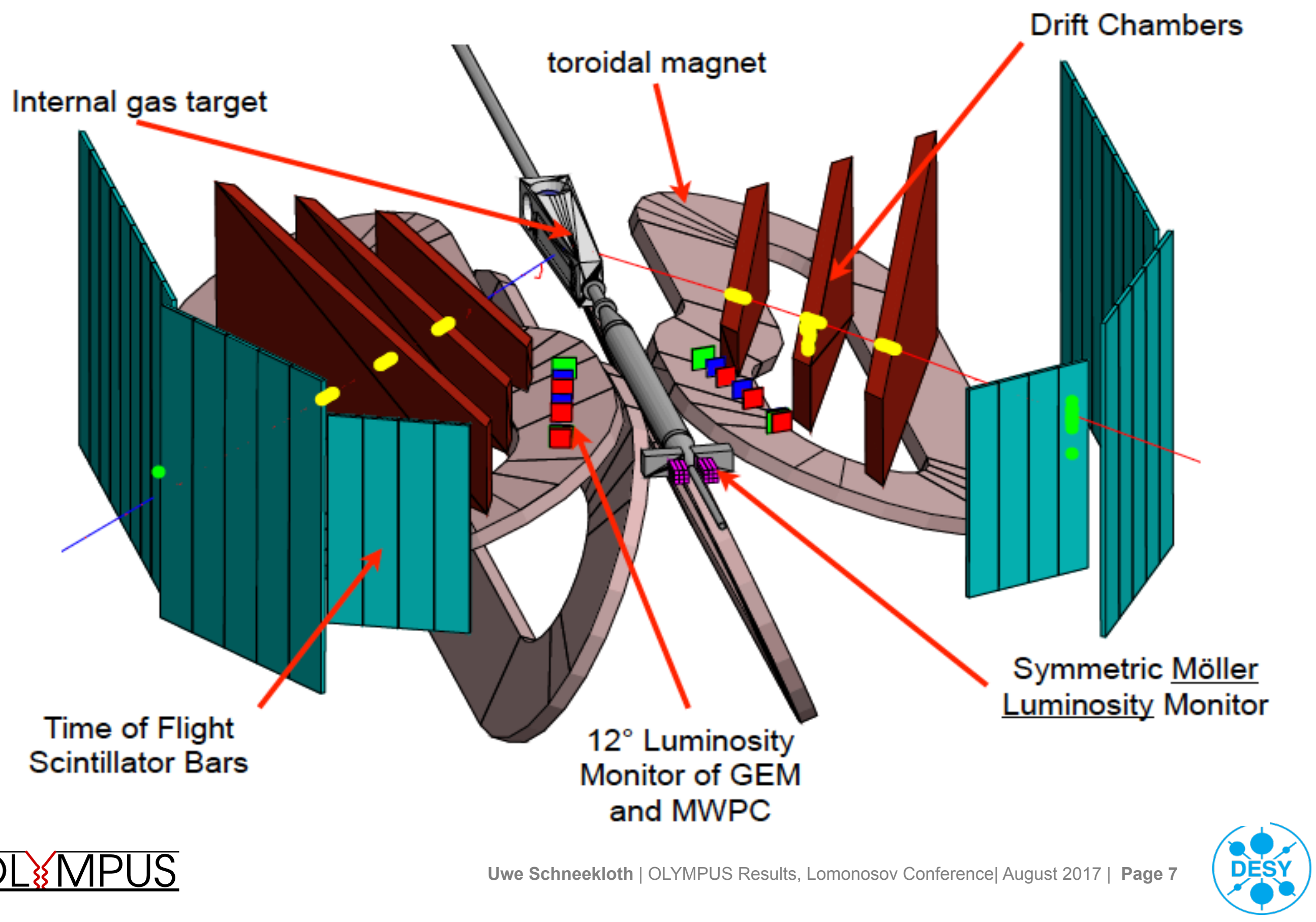




\section{OLYMPUS Detector}

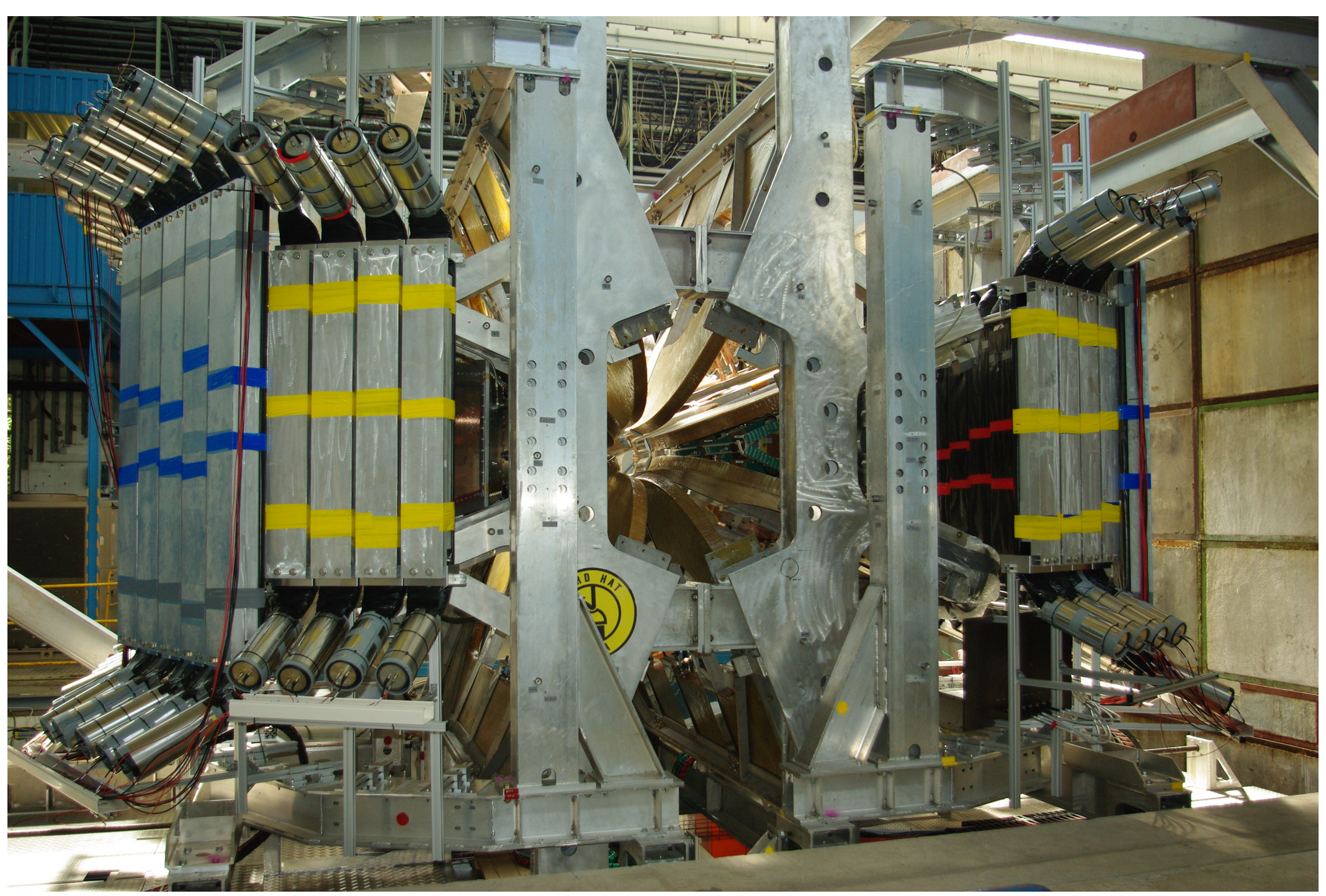

OL $\leq M P U S$ 


\section{DataTaking in 2012}

Limited flow and luminosity in Feb. run

OLYMPUS Luminosity

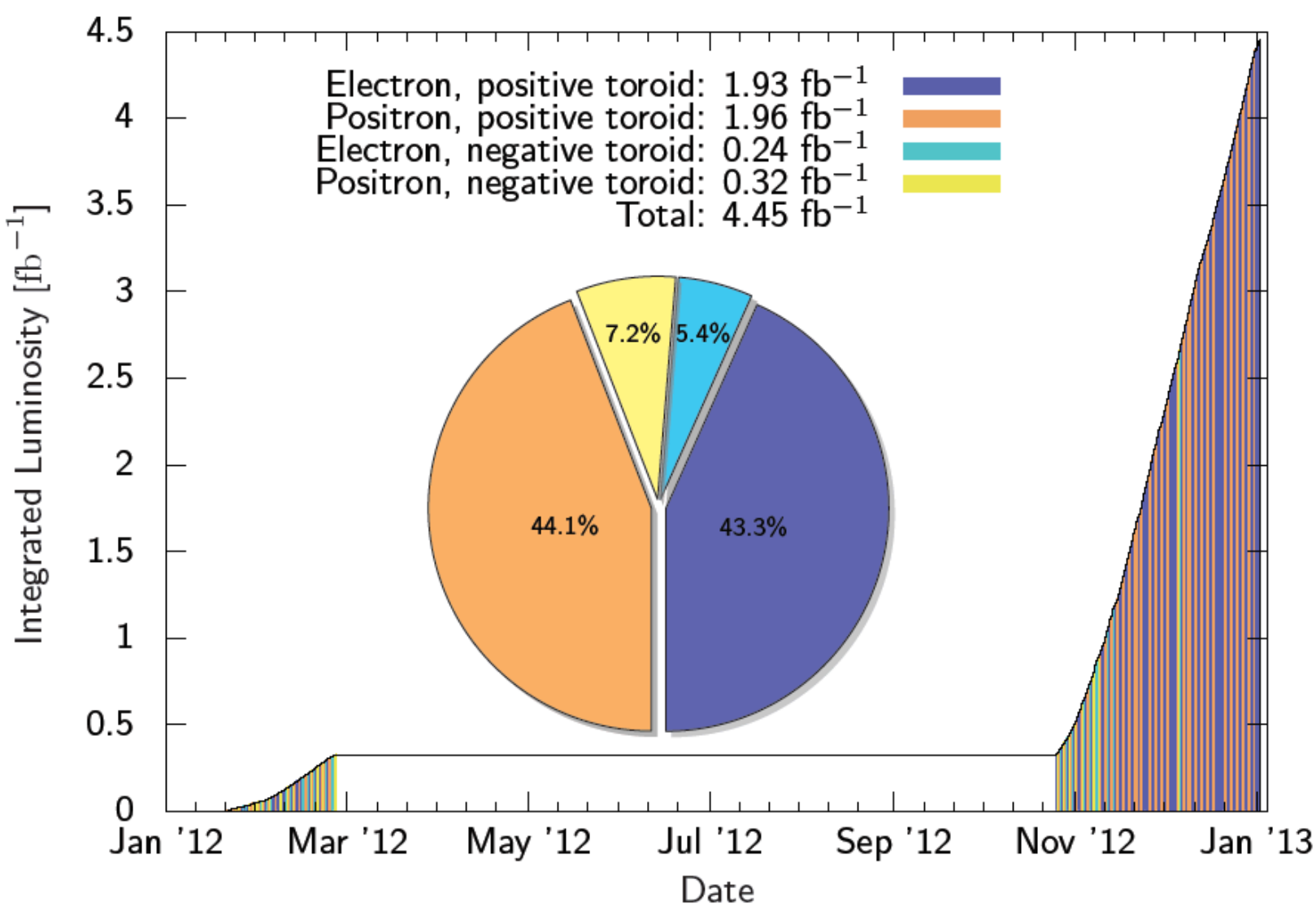

\section{Fall run}

$>$ Full hydrogen flow

$>$ DORIS top-up mode

$>$ Excellent performance

$>$ Exceeded integrated luminosity:

- Design 3.6fb-1, achieved 4.45fb-1

$>$ Daily switch of beam species, good balance

$>$ Mainly positive toroid polarity due to background

$>$ Negative field for systematics checks 


\section{Luminosity Determination}

Three independent measurements

$>$ Slow Control (beam current and target density)

- $2 \%$ between beam species, $5 \%$ absolute

$>$ Tracking telescopes at $12^{\circ}$ (elastic ep scattering at small angles)

- MWPC with coincident proton in WC

- $0.46 \%$ between beam species, $2.4 \%$ absolute

$>$ Multi-interaction events $\left(e^{ \pm} e \rightarrow e^{ \pm} e\right)+\left(e^{ \pm} p\right.$ $\rightarrow \mathrm{e}^{ \pm} \mathrm{p}$ ) at $1.29^{\circ}$ in SYMB monitor

- High statistics measurement, no dead time

- $0.1 \%$ statistical, $0.27 \%$ systematic

Need $e^{+} p / e^{-p}$ luminosity ratio, not precise absolute luminosity

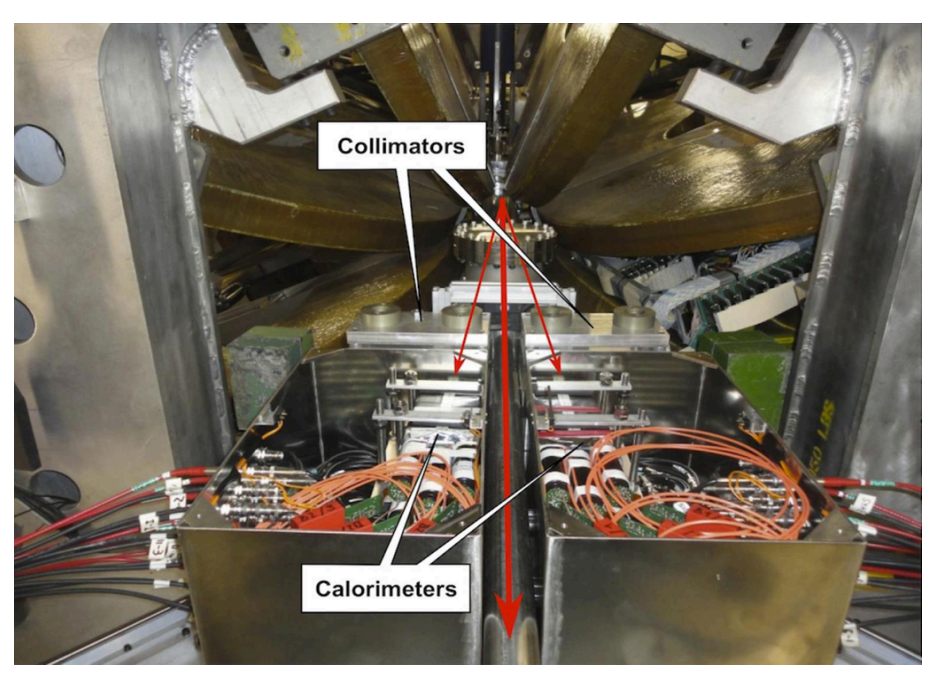

Chose multi-interaction events, most accurate

$>$ Negligible TPE at $1.29^{\circ}$

$$
\text { - }\left\langle Q^{2}\right\rangle=0.002 \mathrm{GeV}^{2}
$$

$>$ Allows measurement of TPE at $12^{\circ}$

- $R_{2 \gamma}=0.9975 \pm 0.010 \pm 0.0053$

- $\left\langle Q^{2}\right\rangle=0.165 \mathrm{GeV}^{2},\langle\varepsilon\rangle=0.98$ 


\section{Radiative Corrections}

Independent elastic $\mathrm{e}^{ \pm} \mathrm{p}$ generators written at MIT (weighted)

$>$ Radiative corrections include:

- Initial and finale state beamsstrahlung for lepton and proton, vertex corrections, vacuum polarization and soft two photo exchange

- Hard two photon exchange not included

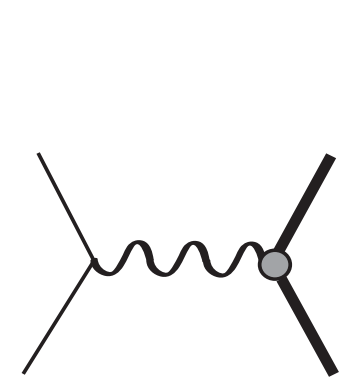

One-photon exchange

\section{soft TPE}

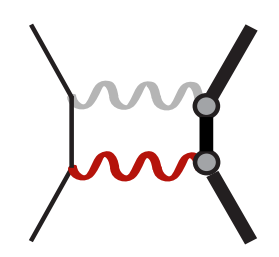

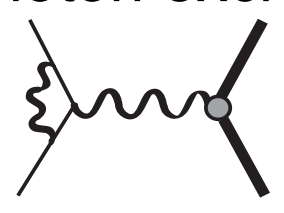

Lepton vertex correction

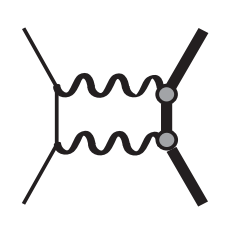

Two-photon exchange

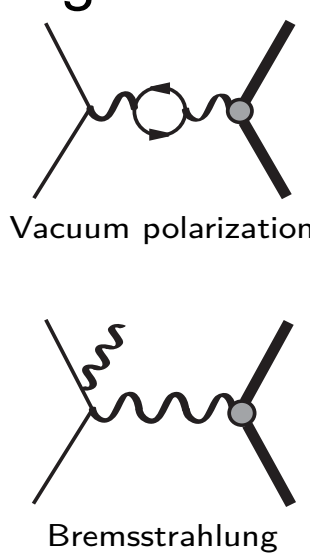

hard TPE
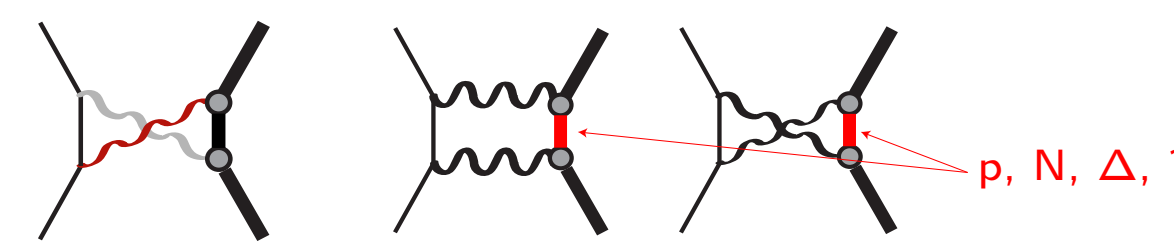

New Møller/Bhabha generator with radiative corrections

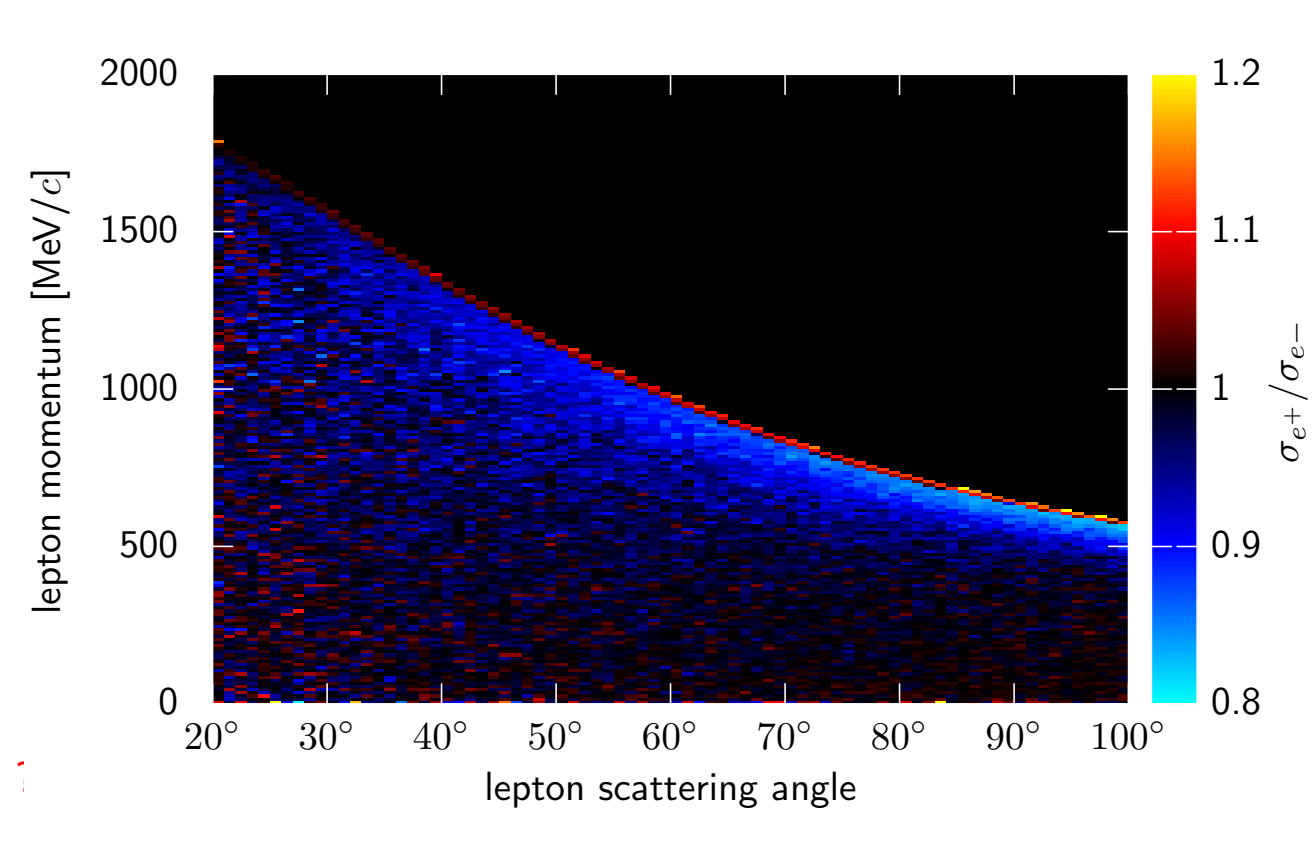

Even powers of $z$ same for $\mathrm{e}^{+}$and $\mathrm{e}^{-}$ scattering, cancel in ratio 


\section{Radiative Corrections Depend on Experiment}

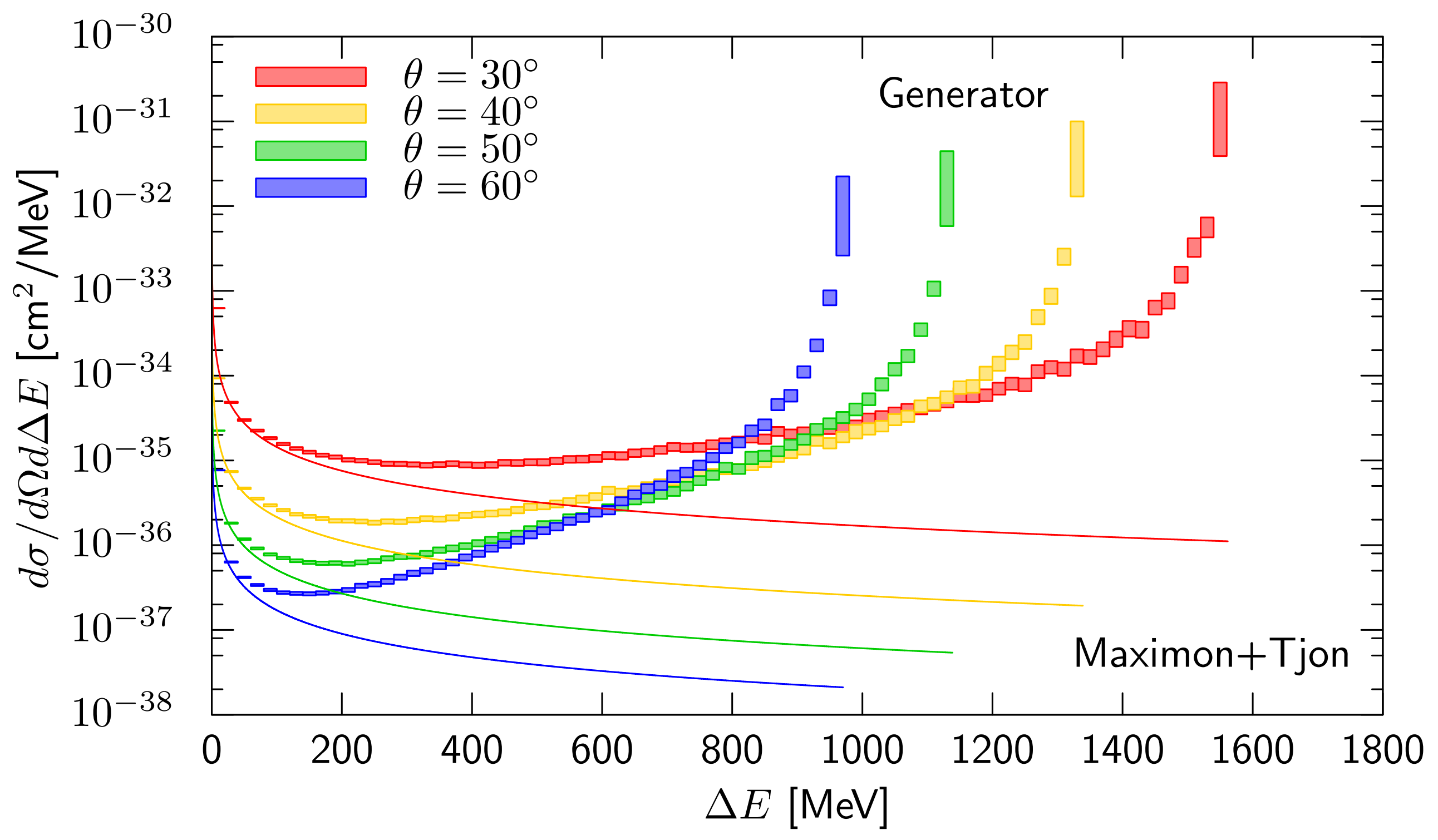

Inelastic

OL 3 MPUS 


\section{Radiative Corrections in OLYMPUS}

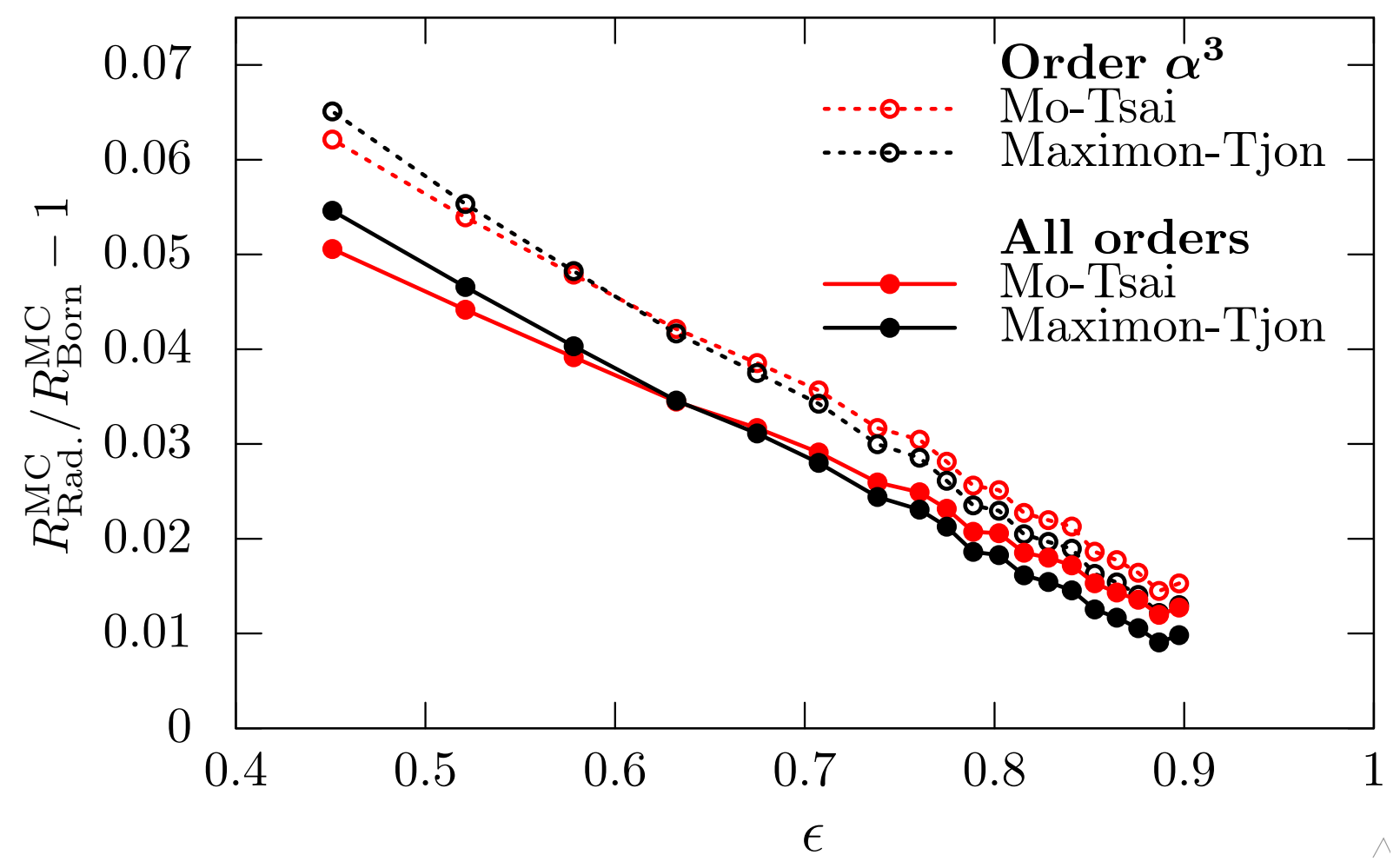

Inelastic 


\section{Schematic of Analysis Procedure}

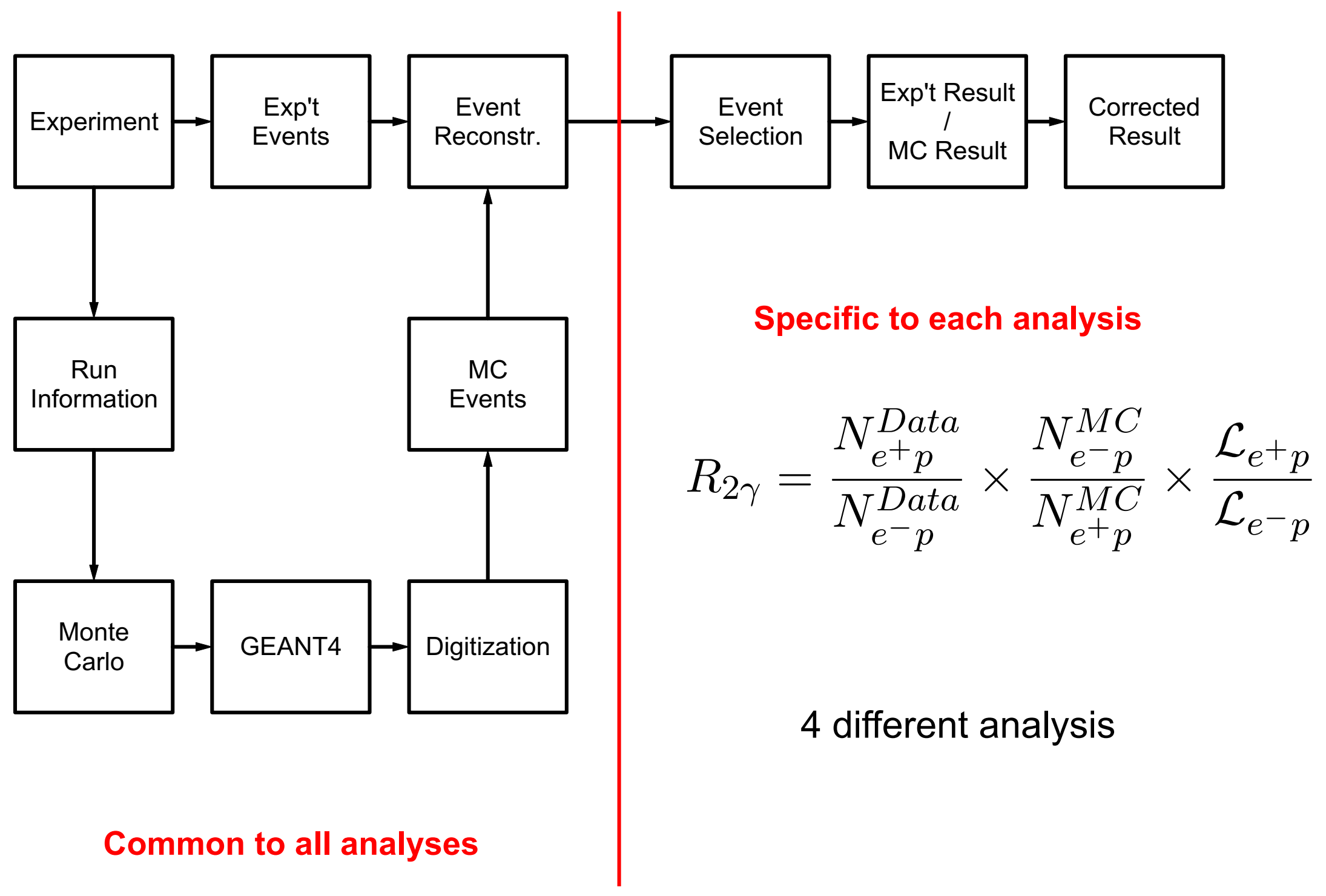




\section{Analysis Procedure}

All analyses share the following:

$>$ Based on same run list and same tracked data files

$>$ Use same tracked, radiatively generated, Monte Carlo files

- Based on same detector calibration, simulation and digitization

$>$ Results normalized with multi-interaction events

$>$ Binned in same $\mathrm{Q}^{2}$ and $\varepsilon$ bins

Analyses are independent in the following:

$>$ Philosophy in selecting elastic candidates vary

$>$ Different order, selection, and size of applying cuts

Four analysis combined for final result

$>$ Results and statistical uncertainty simply averaged

$>$ Variance added to uncorrelated uncertainty in quadrature 


\section{Systematic Uncertainties}

\section{OLYMPUS control of systematics}

$>$ Left / right symmetric detector $\rightarrow$ two independent measurements

$>R_{2 y}$ is a ratio $\rightarrow$ many efficiencies cancel (or reduced)

$>$ Four independent analyses examined and combined

Correlated Systematic uncertainties

$>$ Luminosity (MIE): $0.36 \%$

$>$ Beam energy: $0.04-0.13 \%$

$>$ Beam position and detector position/geometry: $0.25 \%$

$>$ Total: $0.46 \%$

Uncorrelated systematic uncertainties

$>$ Tracking efficiency: $0.25 \%$

$>$ event selection and background subtraction: $0.25-1.17 \%$

$>$ Total: $0.37-1.20 \%$ 


\section{OLYMPUS Results}

Cross section ratio vs. $\varepsilon\left(Q^{2}\right)$

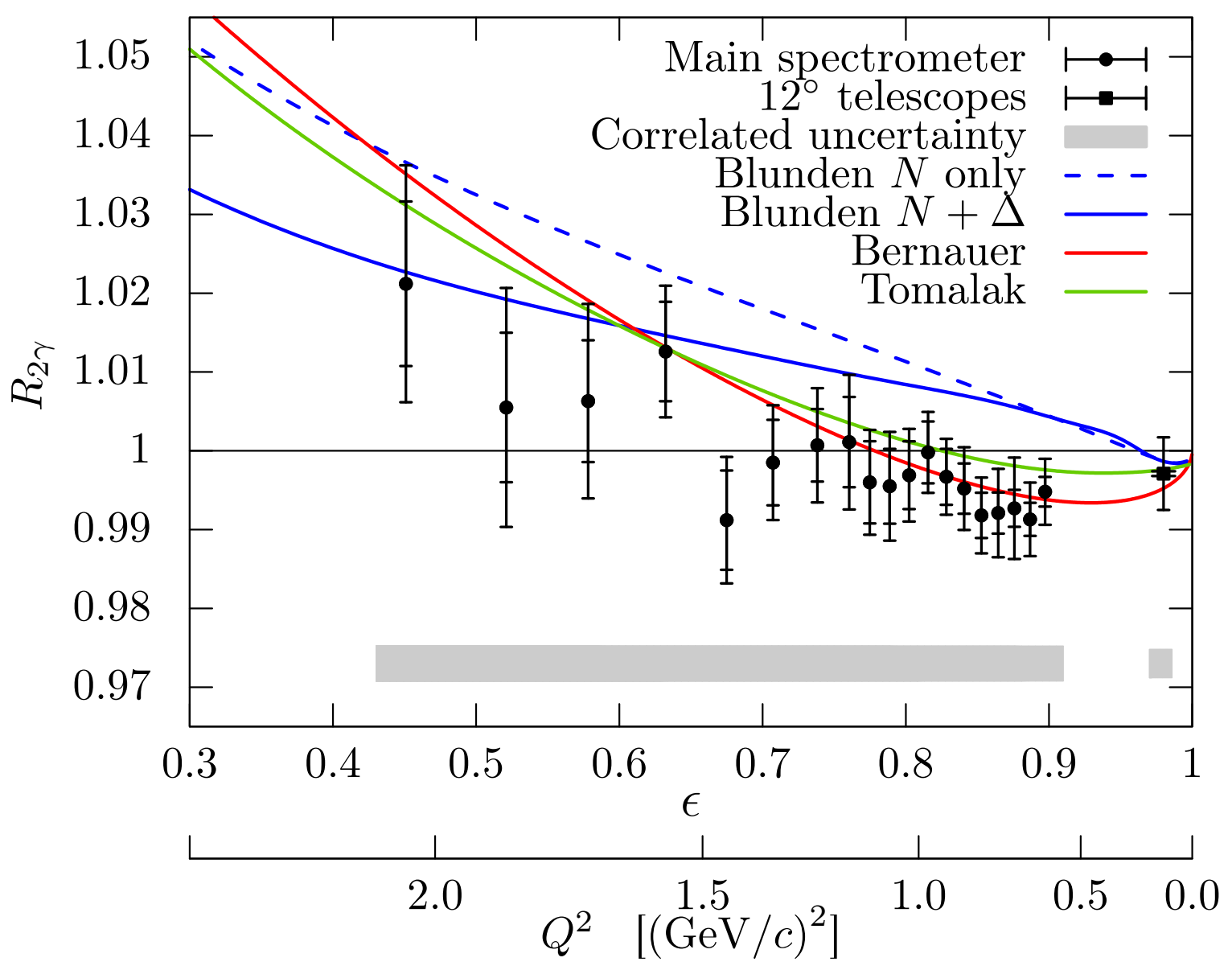

OLYMPUS, B.S. Henderson et al., PRL 118, 092501 (2017)
OLYMPUS results:

$>$ "Hard" two-photon exchange is small, $<1 \%$, at these energies

$>$ Significantly below theoretical calculations

$>$ Reasonable agreement with phenomenological predictions

$>$ Positive slope with decreasing $\varepsilon$ or increasing $Q^{2}$

- Suggest TPE may be present

- May become more important at higher energies 


\section{Kinematic Reach - Recent Measurements}

Kinematic reach $Q^{2}$ vs. $\varepsilon$

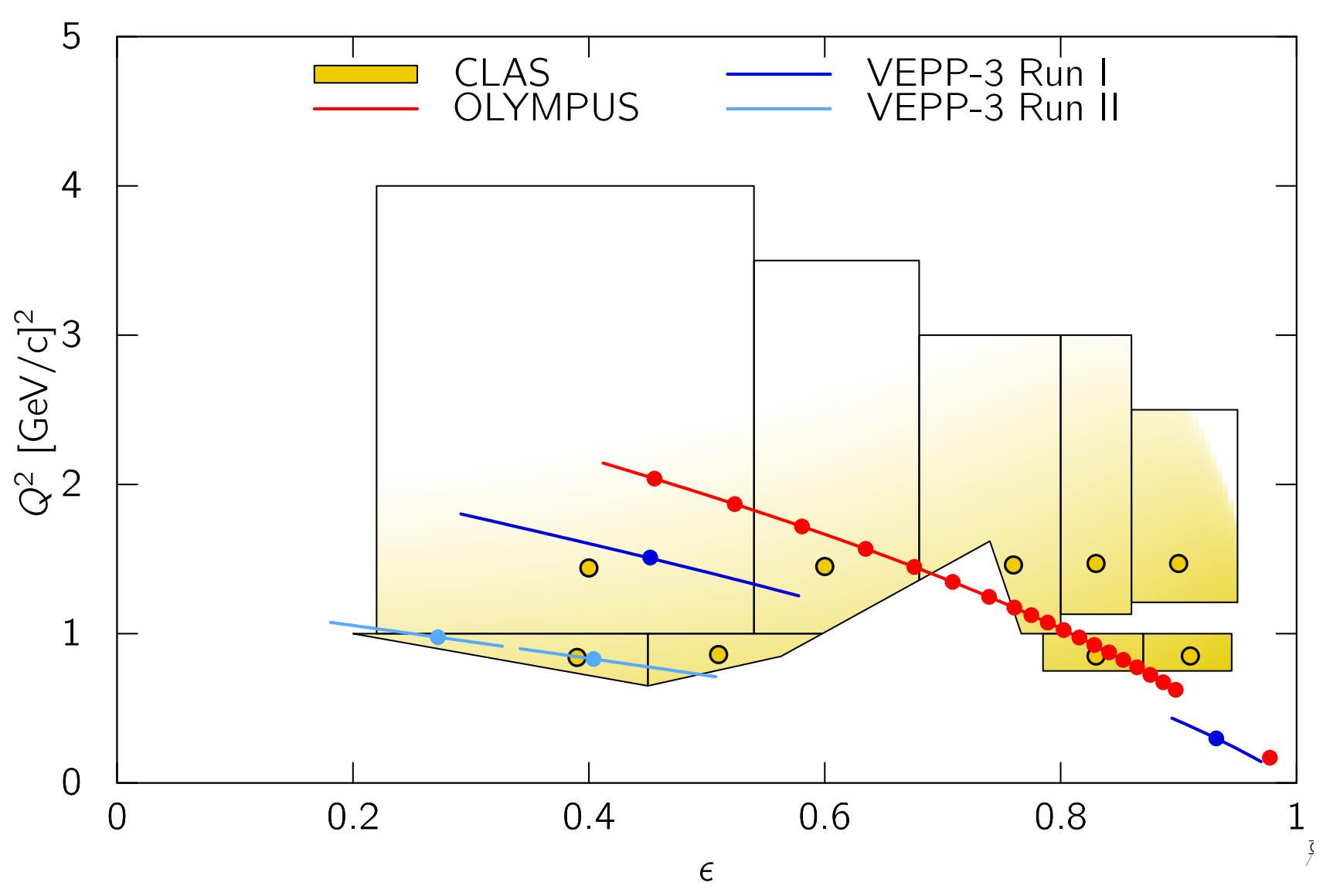

Other recent experiments

$>$ VEPP-3

- E storage ring in Novosibirsk

$>$ CAST

- Fixed target, secondary beam experiment at JLAB

$>$ Comparison of experiments difficult due to different $\varepsilon$ and $Q^{2}$ 


\section{Comparison of Results with Theory}

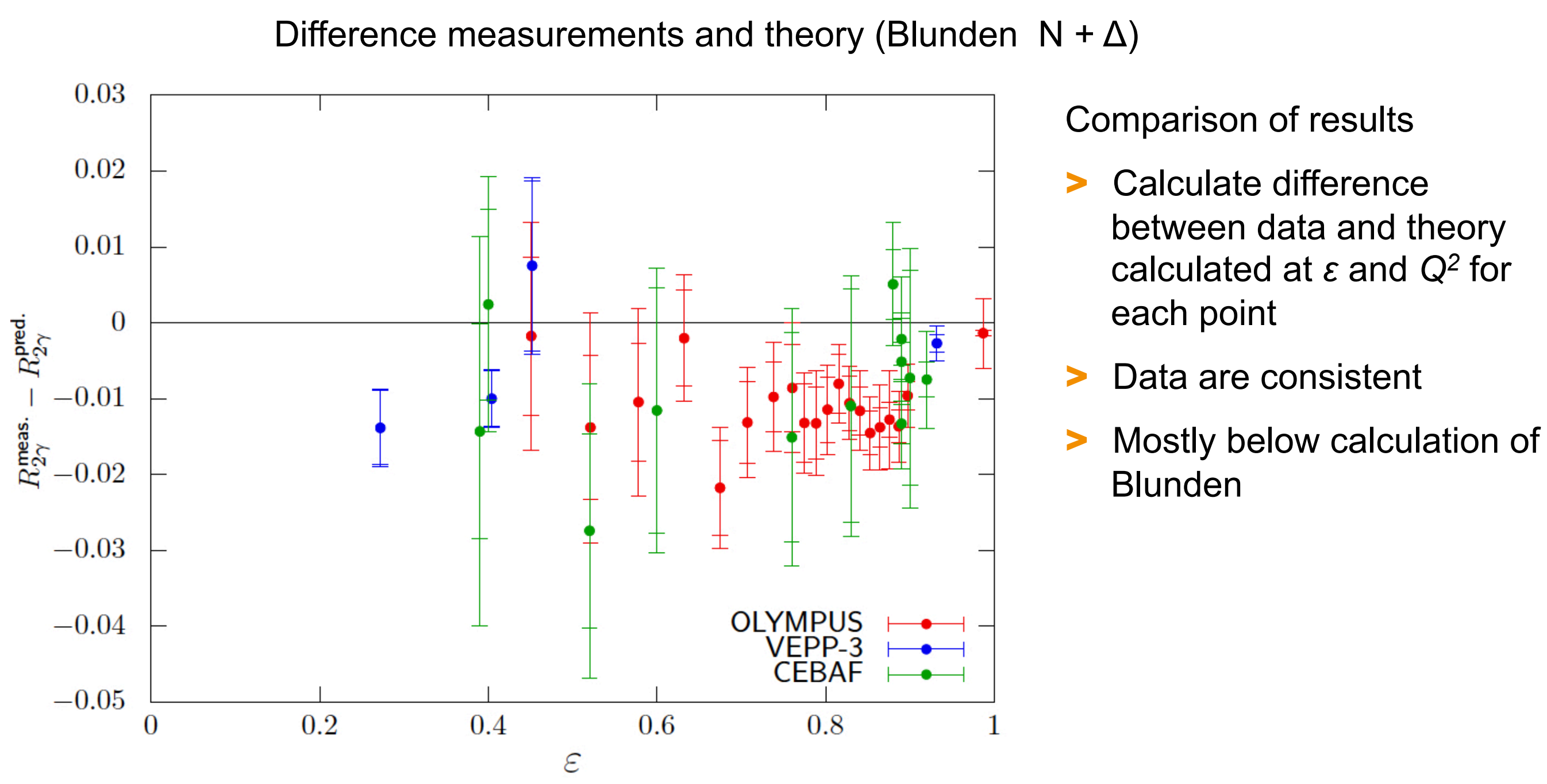




\section{Comparison with Phenomenological Prediction}

Difference measurements and Phenomenological Prediction(J.Bernauer)

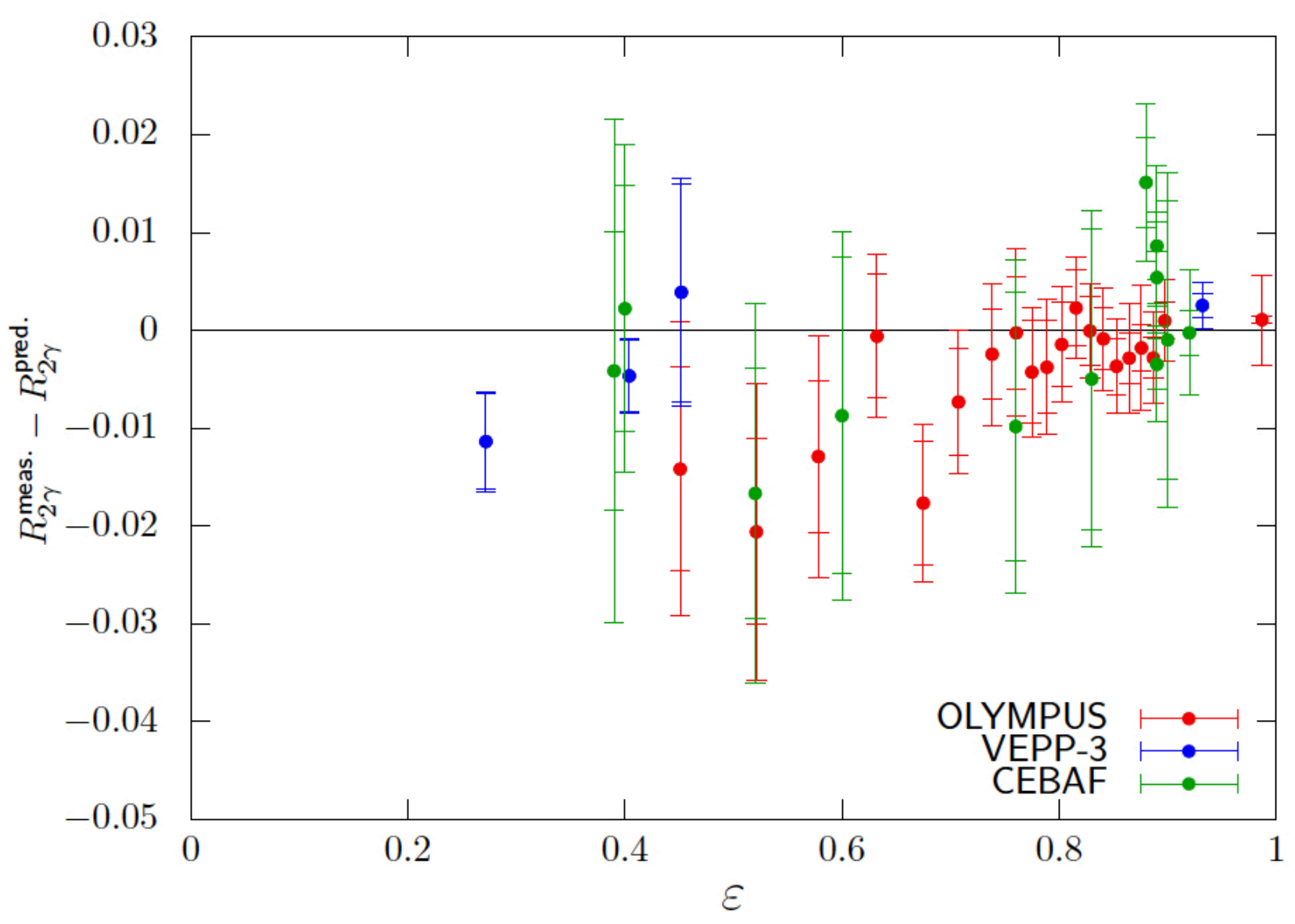

Comparison of results

$>$ Data in good agreement with phenomenological prediction of Bernauer 


\section{Summary of OLYMPUS Results}

$>$ Precision measurement of $R_{2 \gamma}$ for $Q^{2}<2.3 \mathrm{GeV}^{2}$

$>$ Radiative corrections for "soft" TPE important

$>$ Small, $<1 \%$, hard TPE observed

$>$ Evidence for effect increasing with $Q^{2}$

$>$ Results lower than theoretical calculations, but in reasonable agreement with phenomenological predictions

$>$ Further theoretical effort on radiative corrections needed

$>$ Experiments at higher energy required to resolve discrepancy

- Difficult due to rapidly decreasing cross section 


\section{OLYMPUS Collaboration}

Institutes

$>$ Arizona State University, USA

$>$ DESY

$>$ Hampton University, USA

$>$ INFN, Bari, Italy

$>$ INFN, Ferrara, Italy

$>$ INFN, Rome, Italy

$>\mathrm{MIT}$, USA

$>$ Petersburg Nucl. Phys. Inst.

$>$ Universität Bonn, Germany

$>$ University of Glasgow

$>$ Universität Mainz, Germany

$>$ Univ. of New Hampshire, USA

$>$ Yerevan Physics Inst., Armenia 


\section{Backup Slides}

OL $\leq M P U S$ 


\section{OLYMPUS Monte Carlo}

$>$ Utilizing advanced Monte Carlo simulation to account for:

- Beam position/slope

- Detector acceptance/geometry

- Detector resolution and response

- Detector efficiencies

- Radiative corrections (radiative $\mathrm{e}^{ \pm} \mathrm{p}$ and Møller/Bhabha generators developed)

$>$ Recent improvements:

- Refinement of detector geometry model

- Implementation of multiple generator weights for radiative generator systematic studies

- Molecular flow Monte Carlo simulation of target gas flow to improve MC target distribution 


\section{Schedule}

$>2005$

- End of BLAST / Bates experiment

$>2007$

- Letter of Intent

$>2008$

- OLYMPUS proposal

- Conditional approval DESY

$>2009$

- Technical Design Report

- Technical Review

$>2010$

- Approval and funding

- Disassemble Blast detector at MIT ship to DESY,

- Assembly at DORIS, parking position

$>2011$

- Interaction region modified, test experiment

- Detector moved to beam position
$>2012$

- February: first data taking period

- $2^{\text {nd }}$ data taking period Oct. - Dec.

- Exceeded integrated luminosity: design 3.6 $\mathrm{fb}^{-1}$, achieved $4.45 \mathrm{fb}^{-1}$

$>2013$

- Cosmic ray run

- Complete survey

- New magnetic field map

- Beam position monitor calibration

- Reconstruction/data analysis

$>2016$

- Most of analysis finished

$>2017$

- Ratio paper published in PRL 


\section{Target System}

$>$ Internal, windowless gas target

$>60 \mathrm{~cm}$ long storage cell

$>$ Elliptical cross section $(27 \mathrm{~mm} \times 9$ $\mathrm{mm}$ )

$>100 \mu \mathrm{m}$ thick aluminum wall

$>\mathrm{H}_{2}$ flows up to $1 \mathrm{sccm}$

$>$ Cryo cooled $\sim 45 \mathrm{~K}$

$>\mathrm{O}\left(10^{15}\right)$ atoms $/ \mathrm{cm}^{2}$

$>$ Hydrogen produced by generator (electrolysis)

INFN Ferrara, MIT
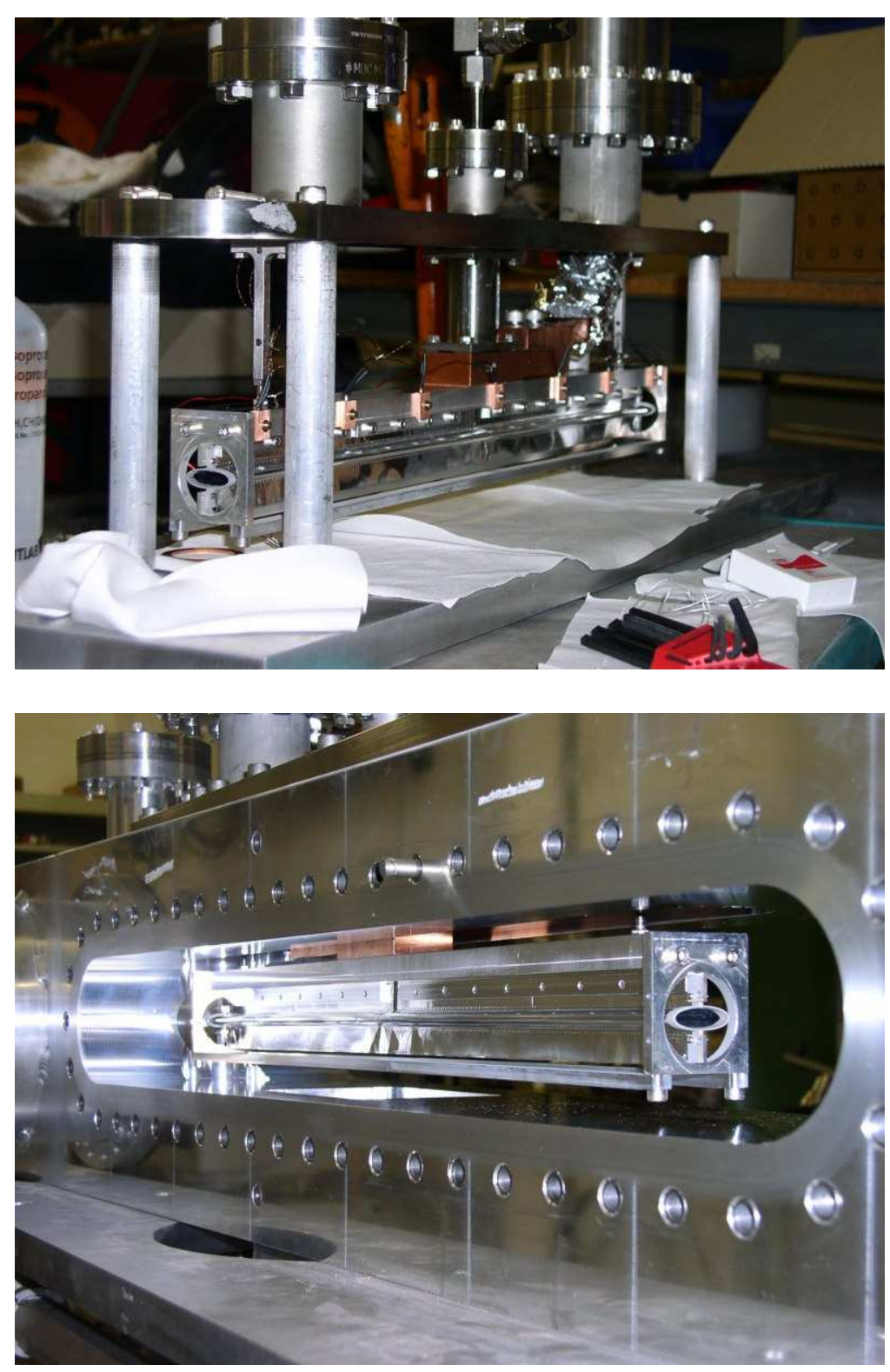

OL 3 MPUS 


\section{Toroidal Magnet}

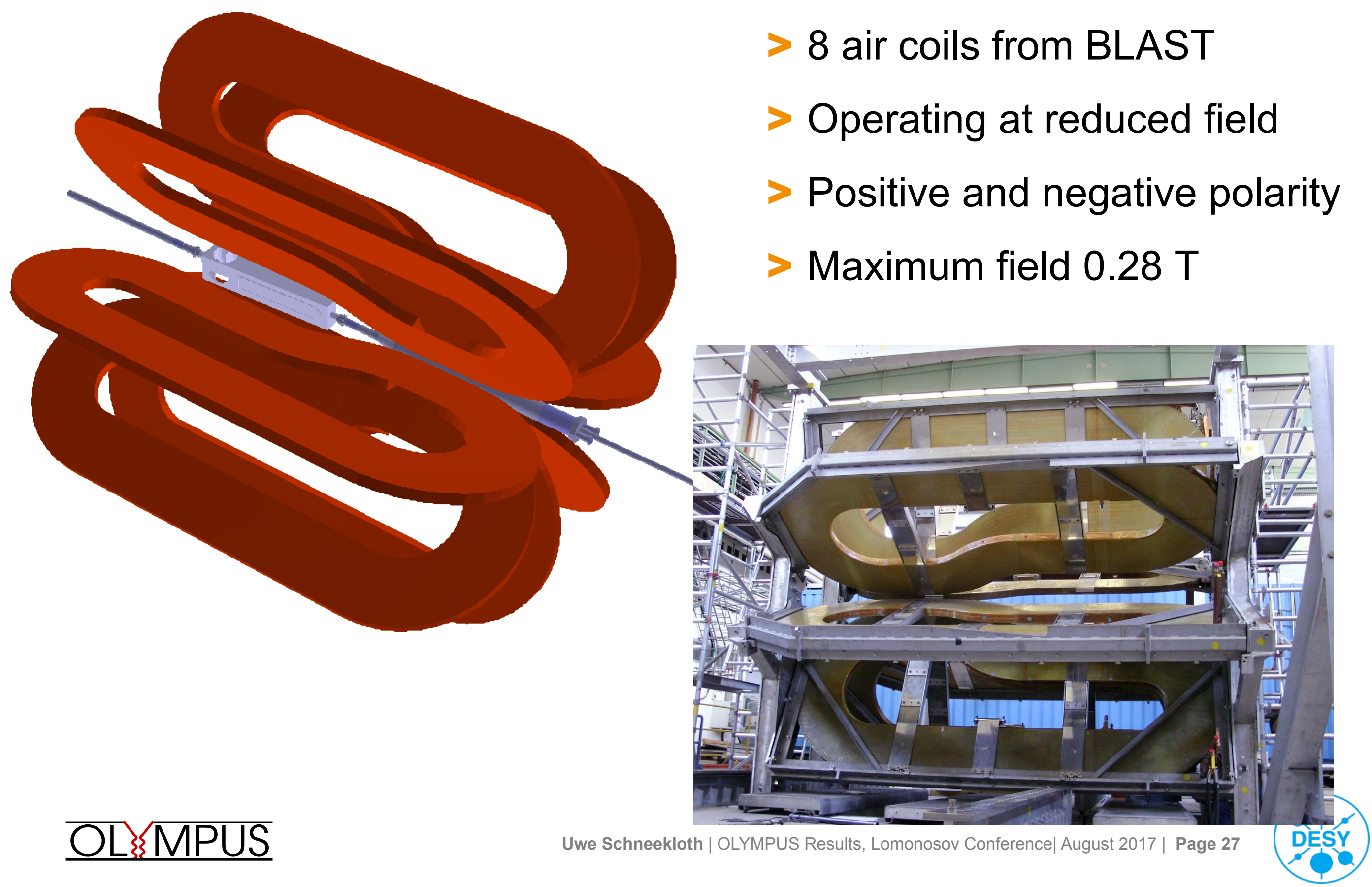




\section{Drift Chambers}

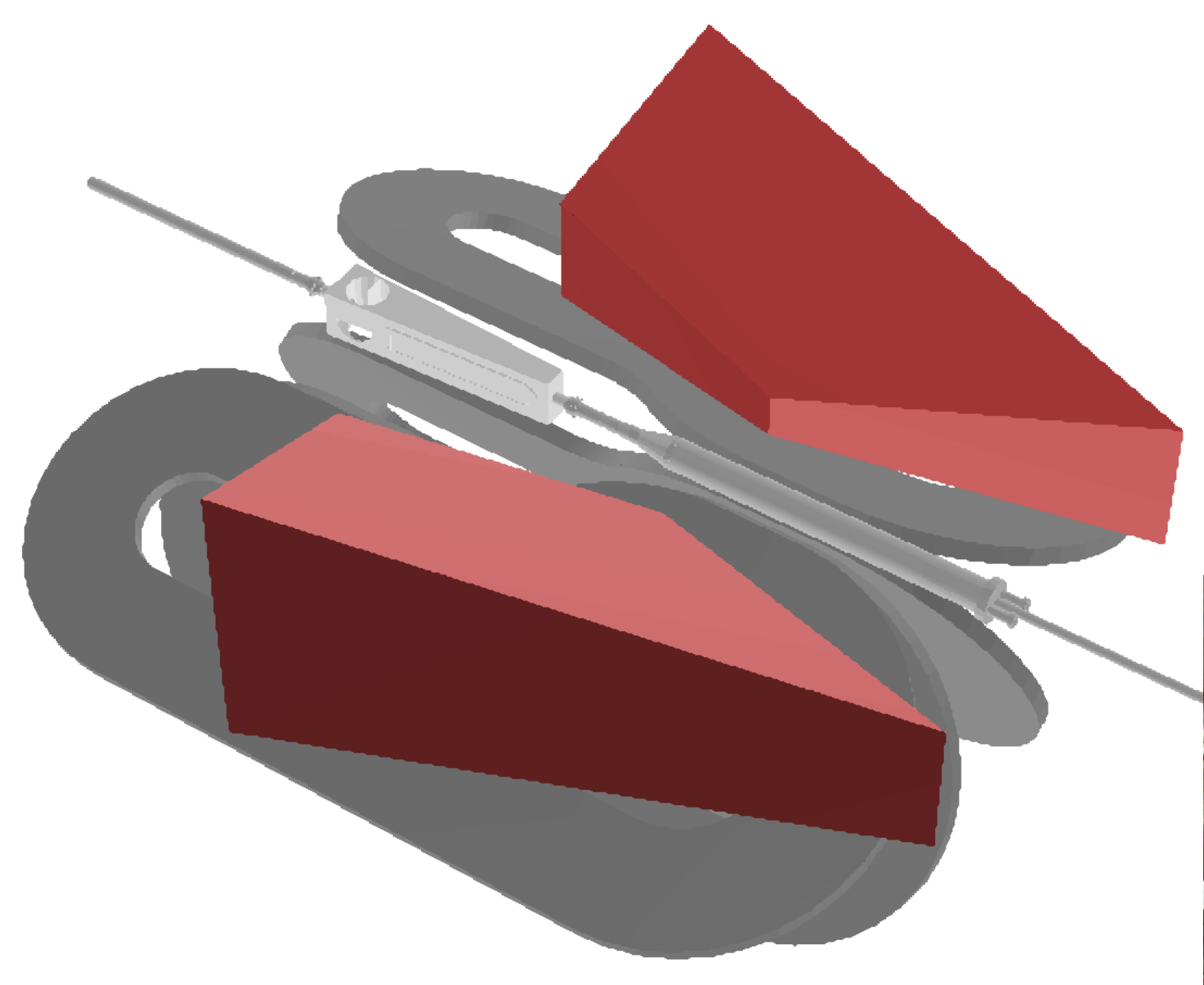

$>$ Two chambers, trapezoidal shape

$>$ Jet-style drift cells

$>5000$ wires each

$>$ Tracks with 18 hits

$>10^{\circ}$ stereo angle

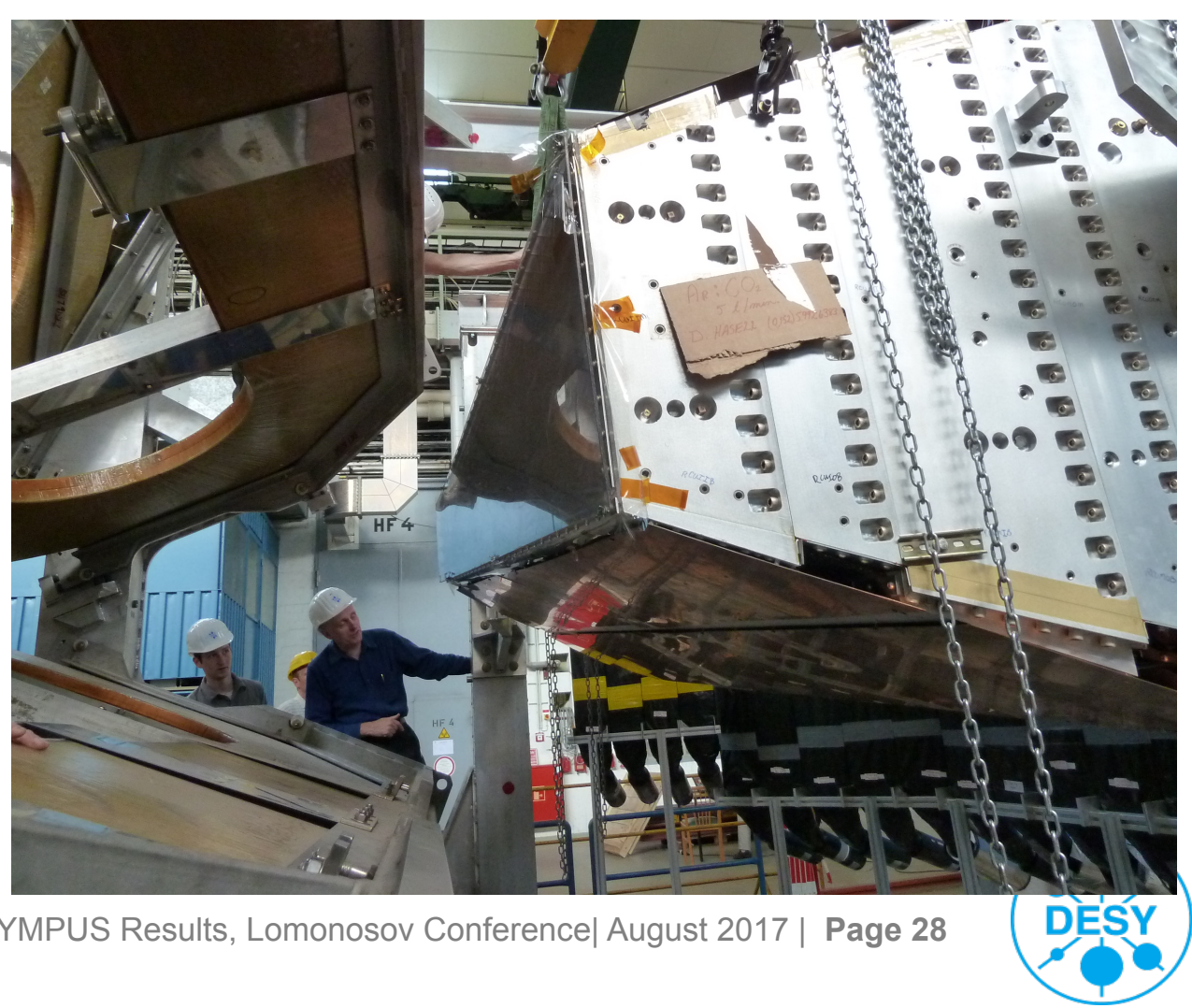




\section{Time - of - Flight Counters}

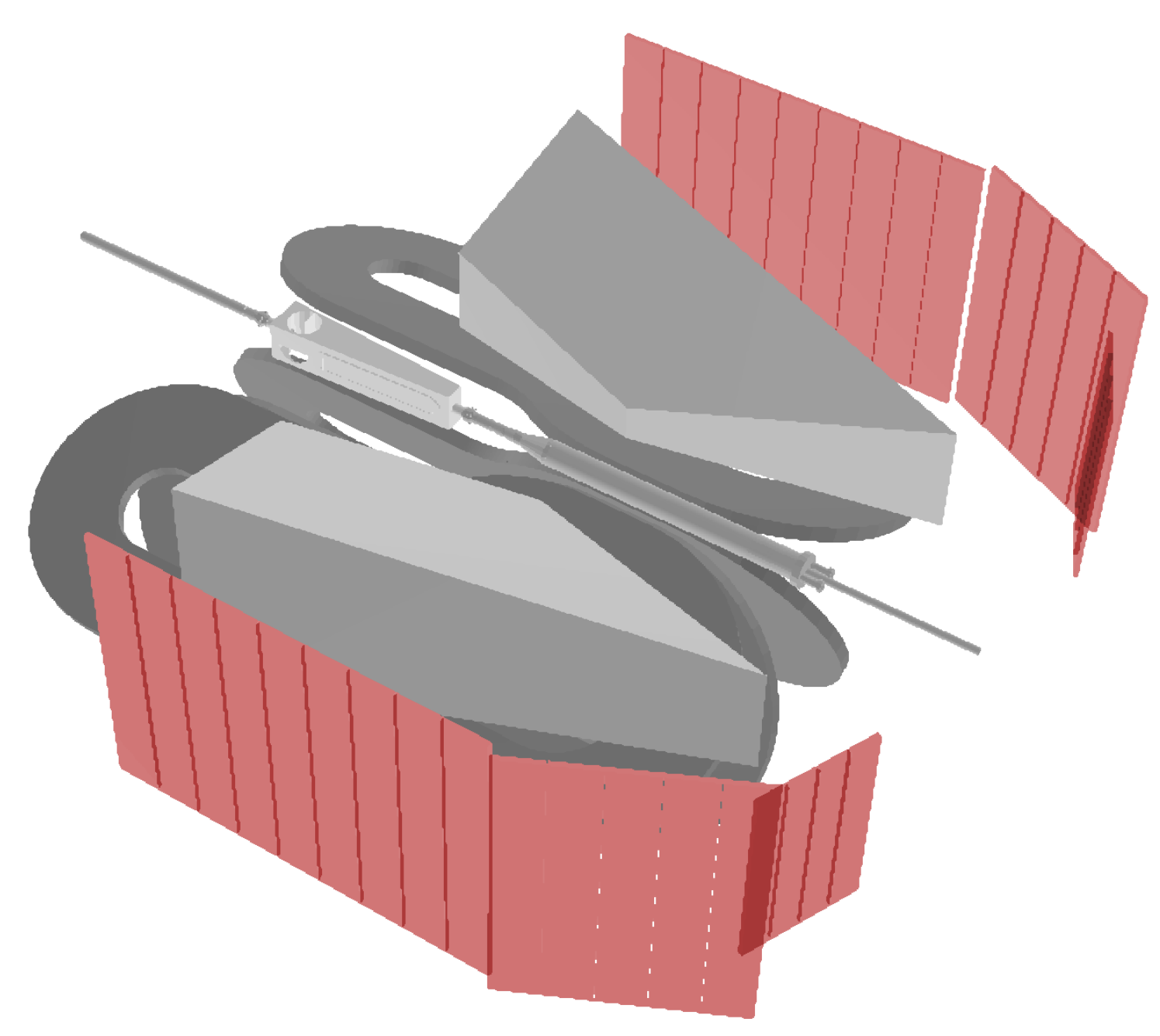

$>$ Scintillation counters from BLAST

$>$ Trigger

- Top/bottom coincidence

- Kinematic constraint

- $+2^{\text {nd }}$ level wire chamber

$>$ Time-of-flight for particle ID 


\section{Target Gas Simulation}

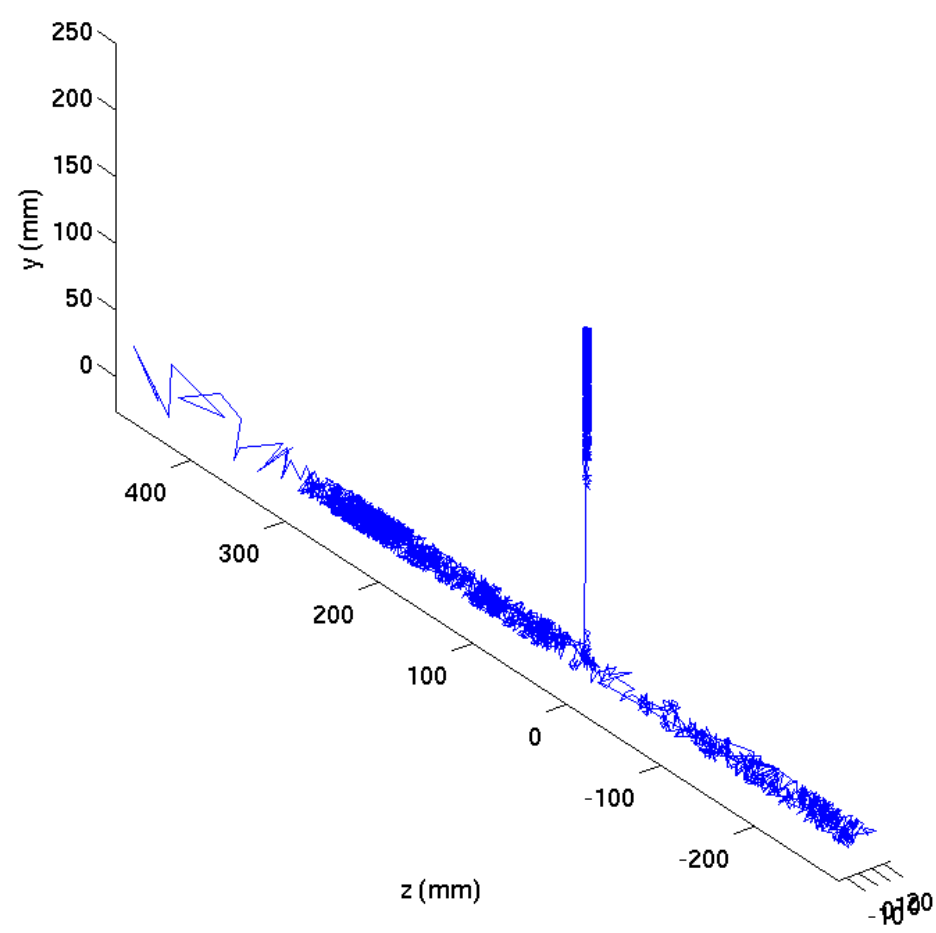

$>$ Molecular flow Monte Carlo simulaton of target more realistic than conductance-based calculation

$>$ Important to get shape of target distribution correct since $\mathrm{e}^{ \pm}$ acceptance can vary along target

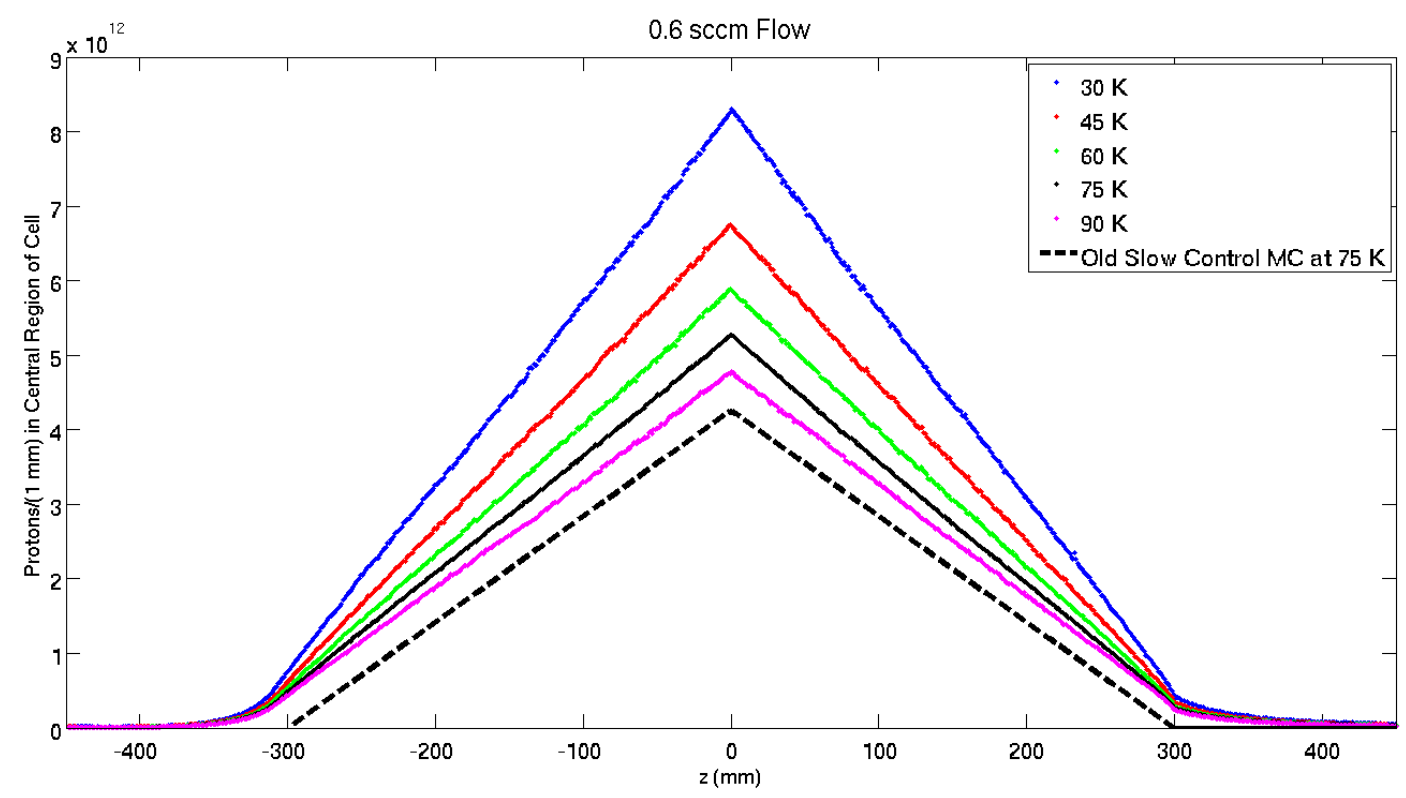




\section{Radiative Corrections in Elastic Cross Section}

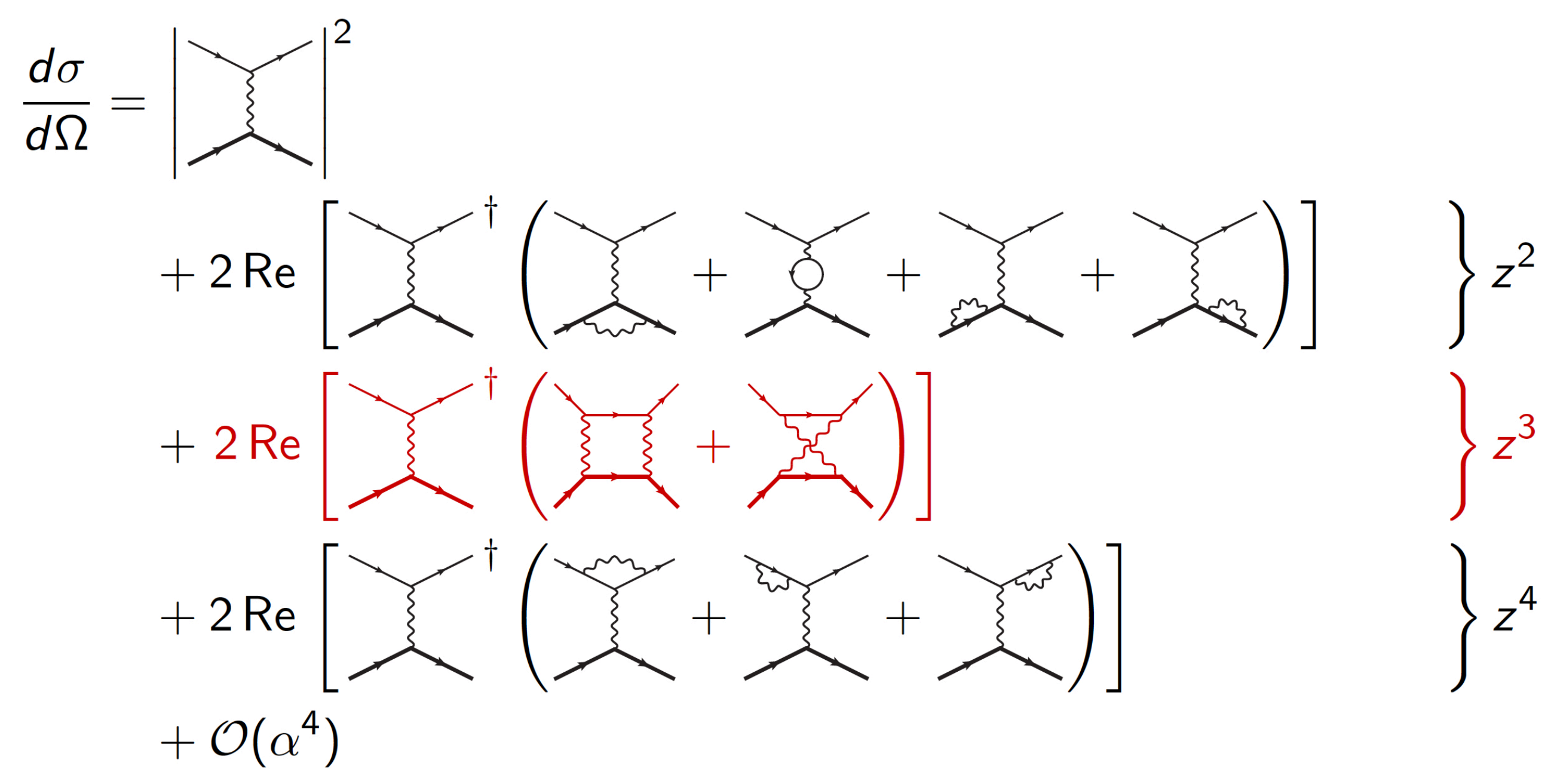

Rebecca Russell, MIT

Even powers of $z$ same for electron and positron scattering

OL $\leq$ MPUS 


\section{Radiative Corrections from Inelastic Processes}

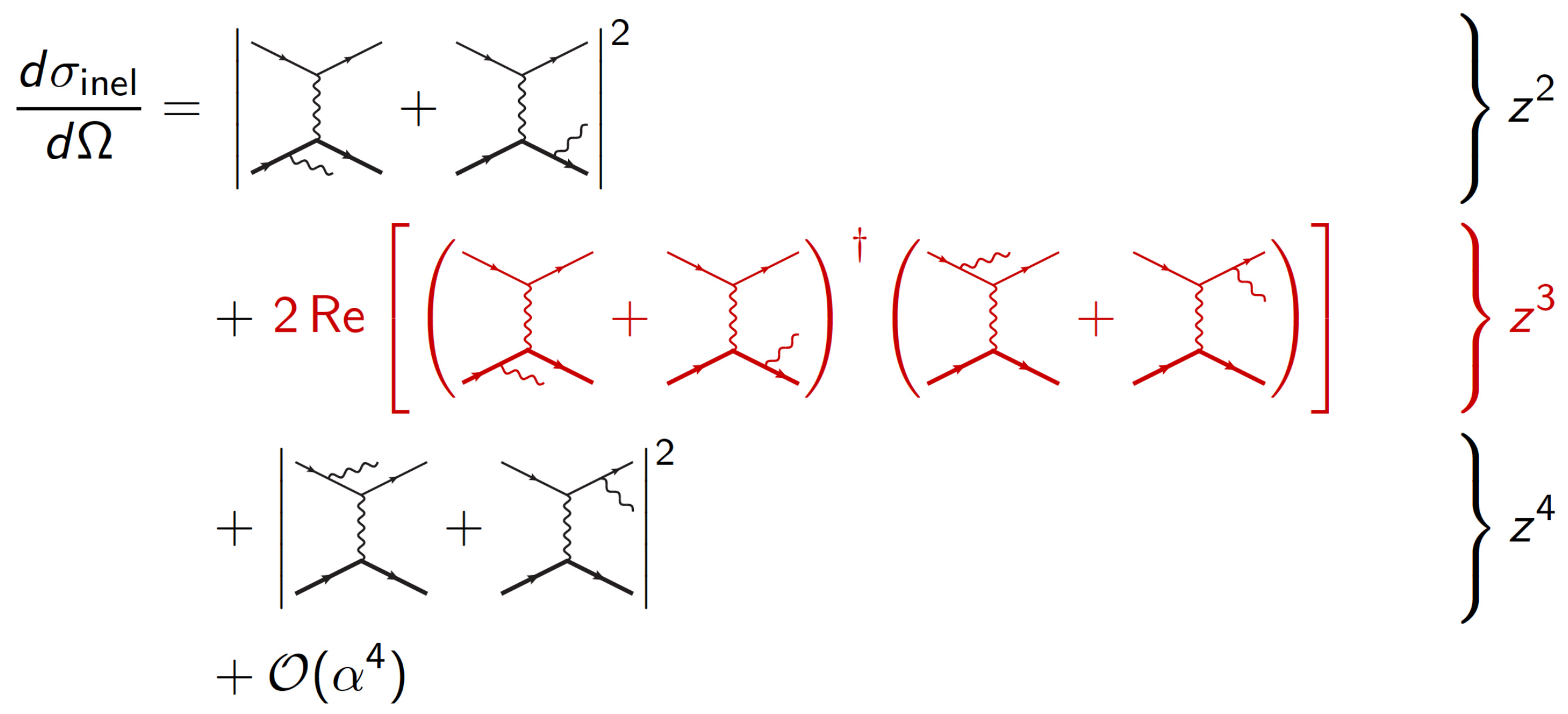

Inelastic IR diverences cancel with elastic divergences

$>$ Must separate "hard" and "soft" parts in TPE

$>$ "soft" part included in radiative corrections, "hard" part measured

$>$ Prescriptions defining "soft" - e.g. Mo, Tsai and Maximon,Tjon

OL 